\title{
Visualization tool for improved accuracy in needle placement during percutaneous radio-frequency ablation of liver tumors
}

\author{
Thomas Stüdeli $^{* a}$, Denis Kalkofen ${ }^{\mathrm{b},}$ Petter Risholm ${ }^{\mathrm{c}}$, Wajid Ali ${ }^{\mathrm{c}}$, Adinda Freudenthal ${ }^{\mathrm{a}}$, Eigil Samset ${ }^{\mathrm{c}}$ \\ ${ }^{\mathrm{a}}$ Faculty of Industrial Design Engineering, Delft University of Technology, Landbergstraat 15, \\ NL-2628 CE Delft, the Netherlands \\ ${ }^{\mathrm{b}}$ Institute for Computer Graphics and Vision, Graz University of Technology, Inffeldgasse 16, \\ A-8010 Graz, Austria \\ ${ }^{\mathrm{c}}$ The Interventional Centre, Rikshospitalet University Hospital, Sognsvannsveien 20, \\ N-0027 Oslo, Norway
}

\begin{abstract}
The European research network "Augmented reality in Surgery" (ARIS*ER) developed a system that supports percutaneous radio frequency ablation of liver tumors. The system provides interventionists, during placement and insertion of the RFA needle, with information from pre-operative CT images and real-time tracking data. A visualization tool has been designed that aims to support (1) exploration of the abdomen, (2) planning of needle trajectory and (3) insertion of the needle in the most efficient way. This work describes a first evaluation of the system, where user performances and feedback of two visualization concepts of the tool - needle view and user view - are compared. After being introduced to the system, ten subjects performed three needle placements with both concepts. Task fulfillment rate, time for completion of task, special incidences, accuracy of needle placement recorded and analyzed. The results show ambiguous results with beneficial and less favorable effects on user performance and workload of both concepts. Effects depend on characteristics of intra-operative tasks as well as on task complexities depending on tumor location. The results give valuable input for the next design steps.
\end{abstract}

Keywords: Visualization, Technology assessment, Intra-operative Imaging, Image-Guided Therapy, Abdominal procedure

\section{INTRODUCTION}

In the percutaneous radiofrequency ablation the surgeon or the intervention radiologist (both will be called interventionist or user) punctures a needle with an electrode (RFA device) through the skin, into the abdomen, into the liver and hits a predefined place in the tumor. After the needle is placed in the tumor, the electrode in the needle is set to dissipate radio-frequency waves and heats up and coagulates the tissue in the neighborhood of the electrode. That means that the tumor is locally destroyed by radiofrequency waves dissipating from the electrode. In most cases the interventionists actually do not use more than a simple needle holder (RFA device) as well as the US image to support the placement of the needle (manual tasks), the final check before the ablation is sometimes done with CT. Poon and colleges [13] report a significant learning curve in their first 100 RFA procedures, although the direct percutaneous approach with local destruction of the tumor is widely seen as a simple and effective way. The accuracy of the needle placement is seen as an important factor for a successful ablation. It might be also one of the reasons for the reported training effect. Many interventionist expressed their wish to be supported by newest technological possibilities during RFA procedures [2], e.g. recently McGahan [10] an experienced interventionist who in 1990, 18 years ago, performed the first RFA of a liver tumor.

\subsection{First design loop}

The European research training network ARIS*ER - "Augmented Reality in Surgery" [3,1] aims to support interventionists with "super vision" (e.g. combined US-CT) and "super sense" (e.g. haptic support for the needle insertion). A result of a first design loop 2005-2006 was an augmented reality system running on a liver phantom, see

*T.P.Studeli@tudelft.nl, phone: +31 1527830 20, http://www.io.tudelft.nl/medisign

Medical Imaging 2008: Visualization, Image-guided Procedures, and Modeling, edited by

Michael I. Miga, Kevin Robert Cleary, Proc. of SPIE Vol. 6918, 69180B, (2008)

$1605-7422 / 08 / \$ 18 \cdot$ doi: $10.1117 / 12.769399$

Proc. of SPIE Vol. 6918 69180B-1 
Figure 1 below. This prototype is based on the software framework "Studierstube" [14] and has been developed at TU Graz with co-efforts of all partners of the consortium. The prototype system runs on a liver phantom and has been assembled at the Interventional Centre in Oslo. It supports the interventionist with some of the newest technological possibilities. The prototype presents the two most widely used image modalities and combines strengths of US- and CTtechnology. The first design of the User Interface (UI) was developed with an idea or technology driven approach. The chosen visualization concept was as open and versatile as possible to be able to investigate on possible display technologies (e.g. HMD). Parallel to this the design problem has been clarified and a medical workflow has been investigated [6].

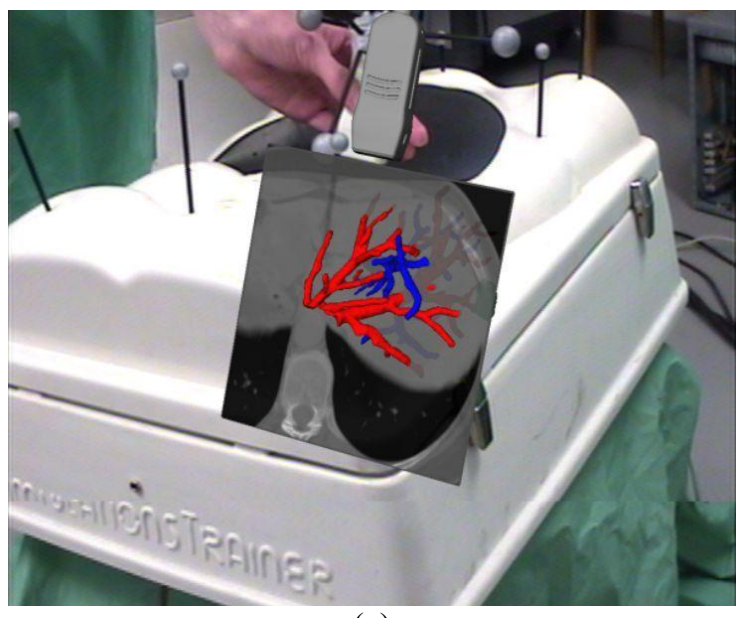

(a)

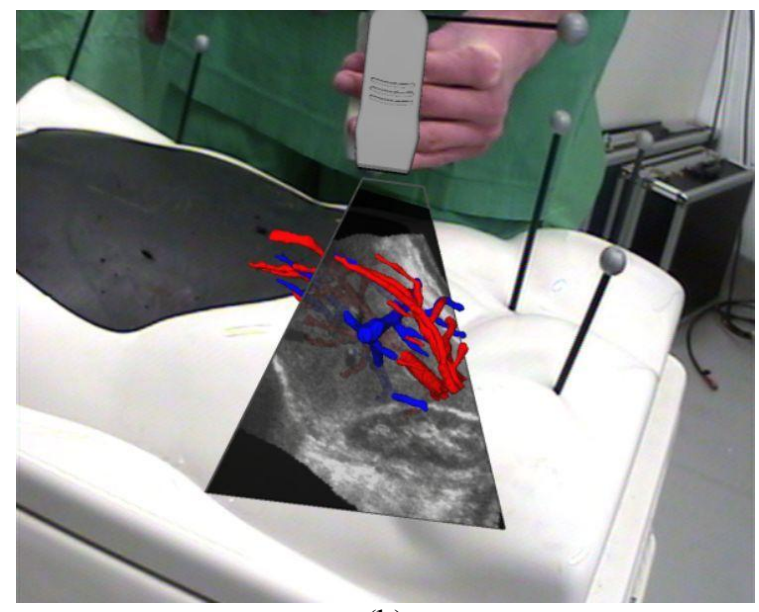

(b)

Fig. 1. Abdominal phantom with prototype of the ARIS*ER RFA system [8]. The hand of the user holds the RFA device (tracked US probe and needle) and hereby controls the display window. The cutting plain is displayed in real-time and virtual information can be added. Here, the hepatic vessel tree is overlaid in red and blue color. The vessel tree is segmented out of pre-operative CT data. (a) On the left side shows CT data displayed in real-time. (b) On the right side shows real-time US data with corresponding segmented vessel tree.

\subsection{Second design loop}

For the actual second design loop (iteration) the aim was to implement and test a visualization concept based on an analysis of the intra-operative tasks and user-medical image interactions. With this approach we aim to face one of the important problems of the RFA procedure the "accuracy of the needle placement" encountered by many users.

Understanding the working system - user, computer system, human-system interactions, environmental aspects, and the working task - is the base of a user interface design process. This applies even more, when the complexity of the working task or the system is increasing. Task analyses are widely used tools in human factors and ergonomics to structure and analyze complex working situation. Cognitive task analysis of needle placements for biopsy, but also other main tasks of interventional radiologists have been done by Johnson and colleagues [7]. They analyzed the classical needle placement procedures without technical support. Their focus laid on training of safe practice for intervention radiologists.

\subsection{Analysis of navigation tasks}

For the design of a computer system to support needle placement an analysis of interventionist's intra-operative tasks during RFA procedure has been performed. It is based on observations, interviews and video assisted cognitive walkthroughs with experienced surgeon and intervention radiologists. The work of the human factors specialist has been gained substantially by the fact that there already was a running prototype. Videos showing the prototype system in use allowed demonstrating the systems functionalities in a comprehensible way. The methodology of these analyses as well as a first rough model how interventionist navigate to the tumor based on cognitive models of navigation and wayfinding and situation awareness have been described earlier [18]. A list of common intra-operative tasks for US- and CT-guided procedures is displayed in Table 1 below. For each intra-operative task basic design concept requirements have been 
derived. The resulting five requirements are based on usability standards, existing models of human orientation, navigation and wayfinding [e.g. 5], as well as on findings from published evaluation studies and user feedback. For the accuracy of the needle insertion (placement) Howard and colleagues e.g. point out the importance of a constant view of the needle tip location as well as deviation of the needle from the predicted path [4].

Table 1. Intra-operative tasks for percutaneous needle placements and corresponding basic design requirements. First and second colon show common interaction tasks and subtasks of interventionists during positioning and insertion of the needle in US-guided and in CT-guided interventions [18]. The third colon indicates corresponding basic design concept requirements.

\begin{tabular}{|c|c|c|}
\hline Interaction tasks & Subtasks & Basic design requirements \\
\hline $\begin{array}{l}\text { Search: Explore abdomen and } \\
\text { liver (sensing environment, } \\
\text { orientation) }\end{array}$ & $\begin{array}{l}\text { - Set-up of a first spatial mental } \\
\text { model of the liver } \\
\text { - Re-detection of the target } \\
\text { tumor(s) }\end{array}$ & $\begin{array}{l}\text { 1. Exploration strategies are } \\
\text { rather individual; the system } \\
\text { should therefore allow } \\
\text { different and individual ways } \\
\text { of exploration. A sufficient } \\
\text { amount of information on } \\
\text { target and context should be } \\
\text { available for the exploration } \\
\text { task. } \\
\text { 2. Detection performance is } \\
\text { correlated with visibility (e.g. } \\
\text { contrast, edges sharpness) of } \\
\text { target. }\end{array}$ \\
\hline $\begin{array}{l}\text { Plan: Placement of the puncture } \\
\text { and exploration of the path } \\
\text { trajectory of the RFA probe } \\
\text { (Spatial orientation, navigation } \\
\text { and wayfinding) }\end{array}$ & 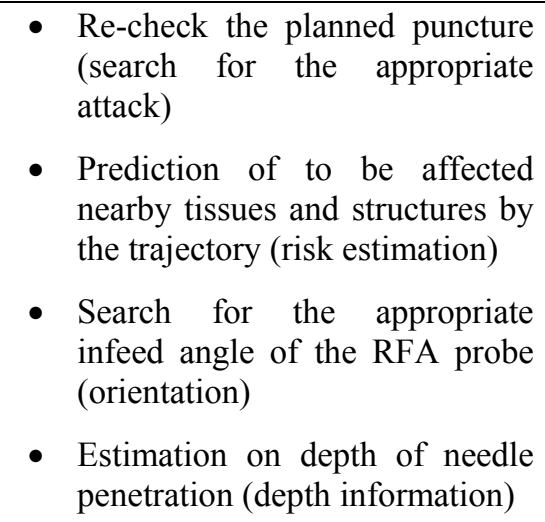 & $\begin{array}{l}\text { 3. Spatial search task and spatial } \\
\text { orientation performance is } \\
\text { better when the user has to } \\
\text { translate only a minimal } \\
\text { amount of spatial information. } \\
\text { 4. Visualization of needle } \\
\text { trajectory plane or path: } \\
\text { information of depth and } \\
\text { affected tissues in the planned } \\
\text { needle trajectory. }\end{array}$ \\
\hline $\begin{array}{l}\text { Act: Action control } \\
\text { insertion of the RFA probe } \\
\text { (movement control) }\end{array}$ & $\begin{array}{l}\text { - } \begin{array}{l}\text { Behavior of tissue structures and } \\
\text { during insertion (deformation } \\
\text { and movements) }\end{array} \\
\text { - Behavior of liver (respiratory } \\
\text { movement, deformation) }\end{array}$ & $\begin{array}{l}\text { 5. Real-time visualization of } \\
\text { needle tip location as well as } \\
\text { deviation of the needle from } \\
\text { the predicted path. }\end{array}$ \\
\hline $\begin{array}{l}\text { Check: Before and during the } \\
\text { ablation the exact position of the } \\
\text { RFA probe referred to the tumor } \\
\text { (accuracy of placement) is } \\
\text { checked and secured (action plan } \\
\text { if necessary). }\end{array}$ & $\begin{array}{l}\text { - Re-detection of the target } \\
\text { tumor(s) on the medical image } \\
\text { - Detection of the needle tip on } \\
\text { the medical image }\end{array}$ & $\begin{array}{l}\text { See requirements above: } \\
\text { 2. Detection performance, and } \\
\text { 4. Information presentation of } \\
\text { needle trajectory }\end{array}$ \\
\hline
\end{tabular}




\subsection{Visualization tool}

Based on these basic design requirements a visualization tool has been sketched at Delft University of Technology. Early design ideas were again checked back with intervention radiologists of Rotterdam MC. The continuous involvement of the user (co-design) guaranteed a focus on the actual use of the tool, always having in mind the expected intra-operative user-medical image interactions.

For this specific design question - "visualization tool for a better accuracy of the needle placement" - the display type had to be specified in the second design loop. This is in contrast to the first prototype, were the requirements still were of more general nature and the visualization concept was open for various display types and UI. The decision was taken to first implement visualization concept for a (widely used) TFT flat screen monitor with high or medium resolution.

The visualization concept aims to support the exploration of the abdomen, planning of needle trajectory, insertion of the needle and checking final needle position in the most efficient way. Main aspects of the visualization concept are the followings:

- The system should provide the user with classical transverse, coronal and sagittal CT slices as well as a 3D overview in parallel.

- The system should be able to display all information in regards to the target tumor. For this the user (e.g. in a preoperative phase) has to indicate to the system the target position of the needle tip in the tumor.

- The system should be able to display relevant segmented information (e.g. rips, liver outline, and hepatic vessel tree) on the CT data (2D) as well as 3D view with.

- The system should present information of the actual needle position in regards to the target and the trajectory line (planned needle path).

Two virtual camera positions, representing different visualization concepts, have been implemented in the ARIS*ER RFA system:

(1) In the external view (Users view camera) the user first chooses with a pointing stick (see Fig. 5, C below) the place in space from where he wants to look at the target tumor. He thereby creates a virtual viewpoint (gaze), the classical CT slices are transformed into a new "external coordinate system": classical transverse into external horizontal view, classical sagittal into external median and classical coronal into an external frontal view. The external horizontal view is perpendicular to the chosen viewpoint and goes through the target tumor, median and frontal are in the direction of the gaze and perpendicular to the horizontal view. In the external view all views remain the same as long as the viewpoint is not changed, see Figures 2(a) \& 3 below.

(2) In the internal view (Needle view camera) the (virtual) viewpoint is fixed at the tip of the needle itself. The user explores the abdomen by pointing the tip of the needle towards the liver and the target tumor and moving over the body surface. Every viewpoint he chooses is at the same time a possible needle trajectory line, he sees exactly the same information in the planning and the subsequent phases. The classical CT slices are transformed into a new and constantly updating "internal coordinate system": classical transverse into internal horizontal view, classical sagittal into internal median and classical coronal into internal frontal view. The internal horizontal view is perpendicular to the orientation of the needle (trajectory line) and goes through the target tumor. The crosslines in the internal horizontal view are a "real targeting" tool. Internal median and internal frontal are in the trajectory of the needle and perpendicular to the internal horizontal view, see Figures 2(b) \& 4 below.

This work describes a first evaluation of the described system with new visualization tool. User performances and user feedback of the two visualization concepts of the tool are compared. The usability study aims to give input for the next design steps of the system as well as the visualization tools. 


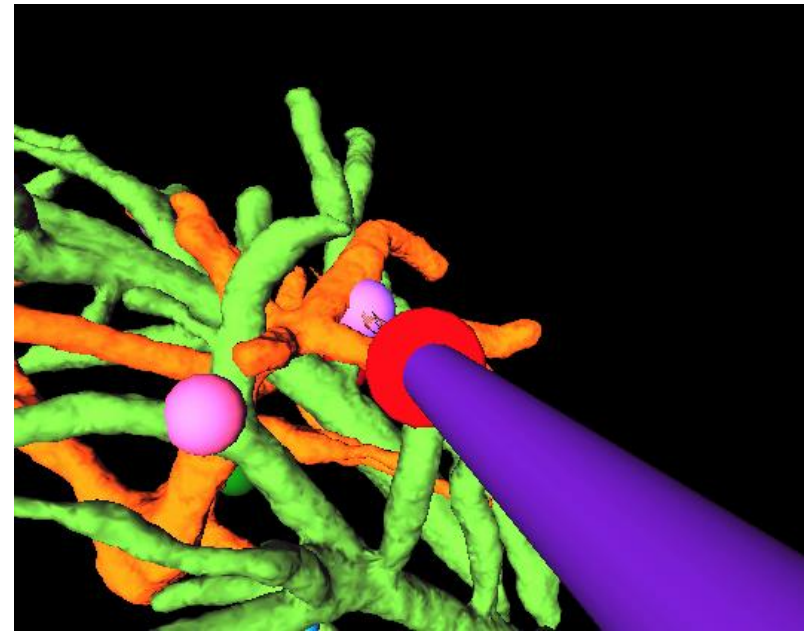

(a)

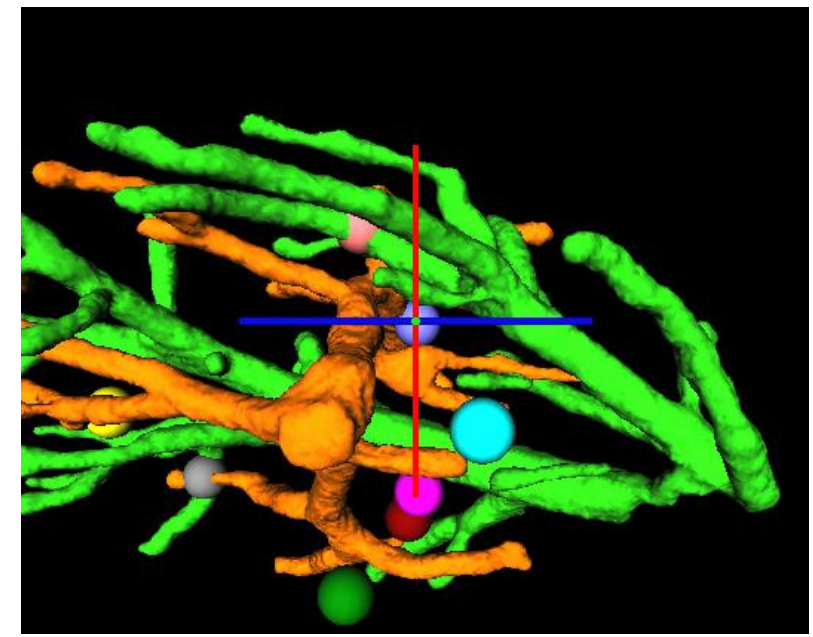

(b)

Fig. 2. The 3D views of the visualization tool. (a) External 3D view shows the needle (dark blue) with a red sphere highlighting the needle tip. The extension of the needle path is indicated with a fine dotted line. (b) Internal 3D view indicates with red-blue crosslines where the needle tip is actually pointing at.

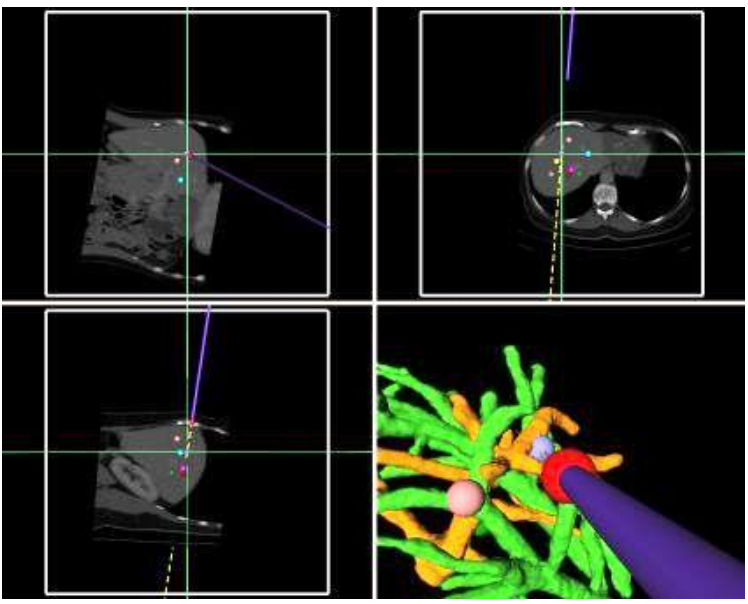

(a)

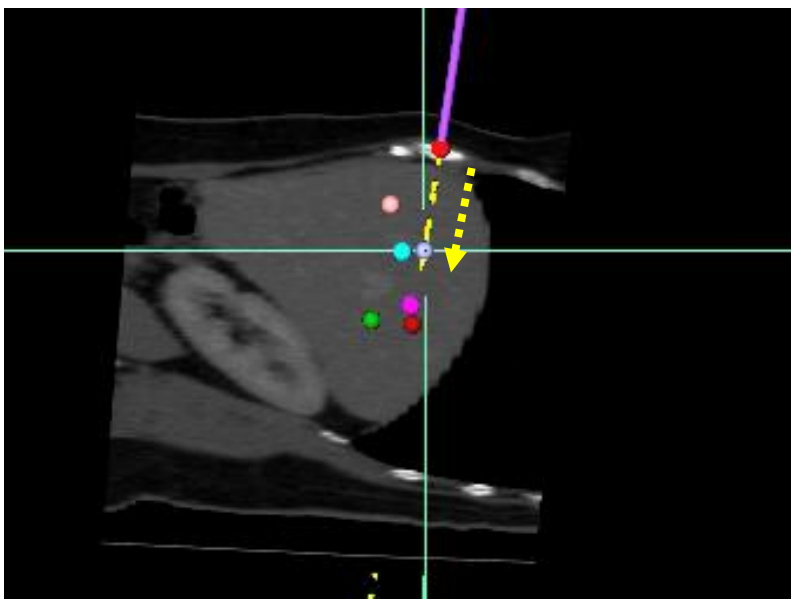

(b)

Fig. 3. Design elements of the external view. (a) Screenshot with all four windows. They show external horizontal view (upper left), external median view (upper right), external frontal view (lower left) and external 3D view (lower right, see also Fig. 2a). The target tumor is central in all 2D views. The needle is represented in blue with a highlighted needle tip (red sphere) and a dotted needle extension (not good visible). (b) Zoomed cut-out of the external frontal slice (down left corner in (a)). In this view the target tumor in the center of the crosslines, the needle is represented in blue with a highlighted needle tip (red sphere), moves along the yellow dotted needle extension, here additionally highlighted with a yellow arrow (not implemented in the system). Along the yellow dotted needle extension penetration depth, tissues and structures on the trajectory can be explored (e.g. risk estimation). 


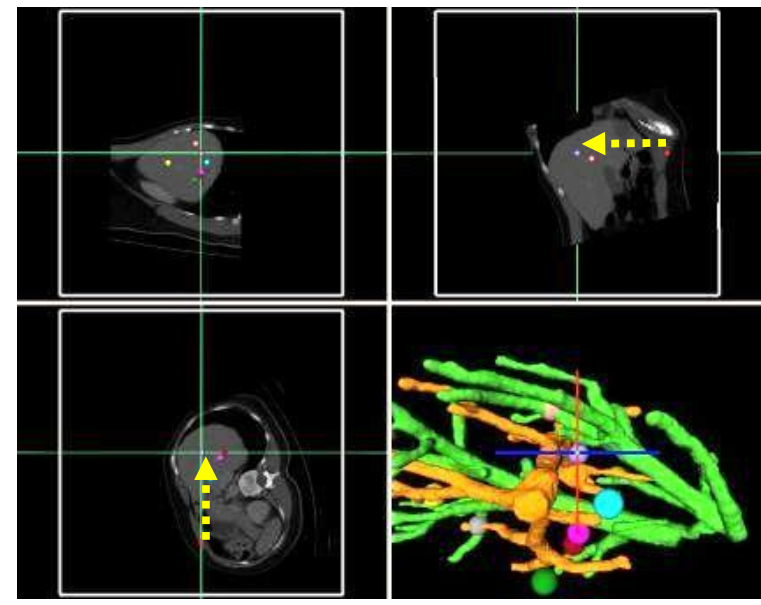

(a)

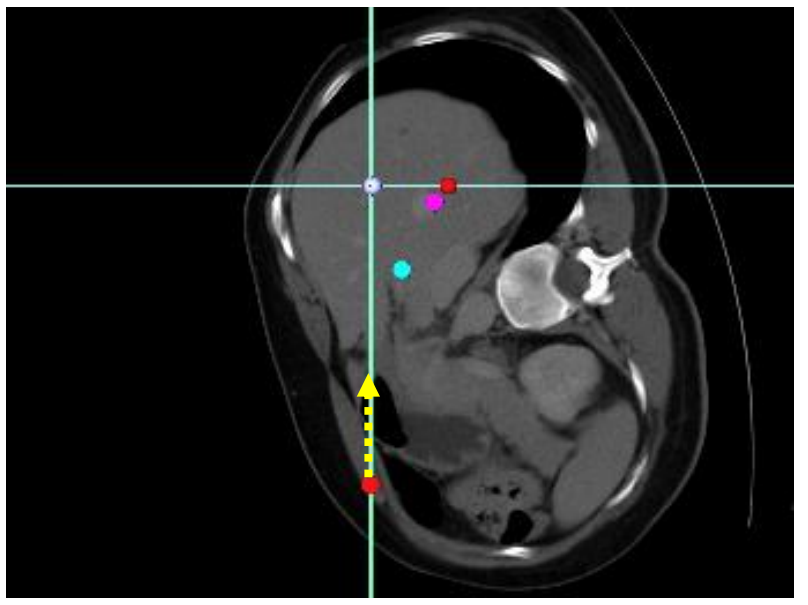

(b)

Fig. 4: Design elements of the internal view. (a) Screenshot with all four windows. They show internal horizontal view (upper left), internal median view (upper right), internal frontal view (lower left) and internal 3D view (lower right, see also Fig. 2b). The needle, indicated with a red sphere, moves along the vertical crossline upwards (frontal slice) and along the horizontal crossline (median view) leftwards, both towards the horizontal view, here highlighted with a yellow arrow (not implemented in the system). (b) Zoomed cut-out of the internal frontal slice (down left corner in (a)). In this view the needle moves along the vertical crossline upwards, here highlighted with a yellow arrow (not implemented in the system). The needle tip is highlighted with a red sphere. Along the crossline penetration depth, tissues and structures on the trajectory can be explored (e.g. risk estimation).

\section{METHODS}

A pilot study with technical subjects has been performed at the TU Graz, Austria to test the experimental setup. The final study at the Interventional centre, University hospital Oslo, Norway took place between November 27 and December 3 2007. Ten male subjects (including ES and PR), age 28 to 54 (35.1 \pm 8.4 years) were recruited among the ARIS*ER team and members of the Interventional centre. All subjects gave their written informed consent and were not reimbursed for participating. The experimental session took approximately one hour in total. It consisted of one session for each subject.

\subsection{Material}

For the simulation study 8 virtual spherical tumors of $1 \mathrm{~cm}$ in diameter were placed in a CT dataset. Tumor locations in the liver were chosen in regard to be realistic tumor locations for percutaneous RFA procedures. Tumor places were chosen that were not too central but also not too peripheral. In order to better identify the different tumors, they were color coded (grey, yellow, pink, blue, brown, purple, green, and red).

The abdominal phantom used the cabinet of a surgical trainer "SimulationTrainer" with its neoprene skin (Fig. 5). For more realistic forces during needle insertion the phantom was filled with floral foam (OASIS® Wet Floral Foam Bricks (http://www.smithersoasis.com/). For this purpose nine bricks (brick size of $23 \times 12 \times 8 \mathrm{~cm}$ ) have been clued together.

Optical markers were placed on the abdominal phantom, needle and pointing stick (Fig. 5). We used RITA StarBurst ${ }^{\mathrm{TM}}$ XL from Rita medical (http://www.ritamedical.com/products/starburstxl.shtml ) as RFA device (needle). After mounting the markers, it offered needle introductions of max. $21 \mathrm{~cm}$ length, the part of the needle that can not be introduced (handle and tracking targets) is $25 \mathrm{~cm}$ (Fig. 5). For the tracking of the phantom, needle and pointing stick the ART tracking system (http://ar-tracking.eu/index.php) has been used with four cameras. 


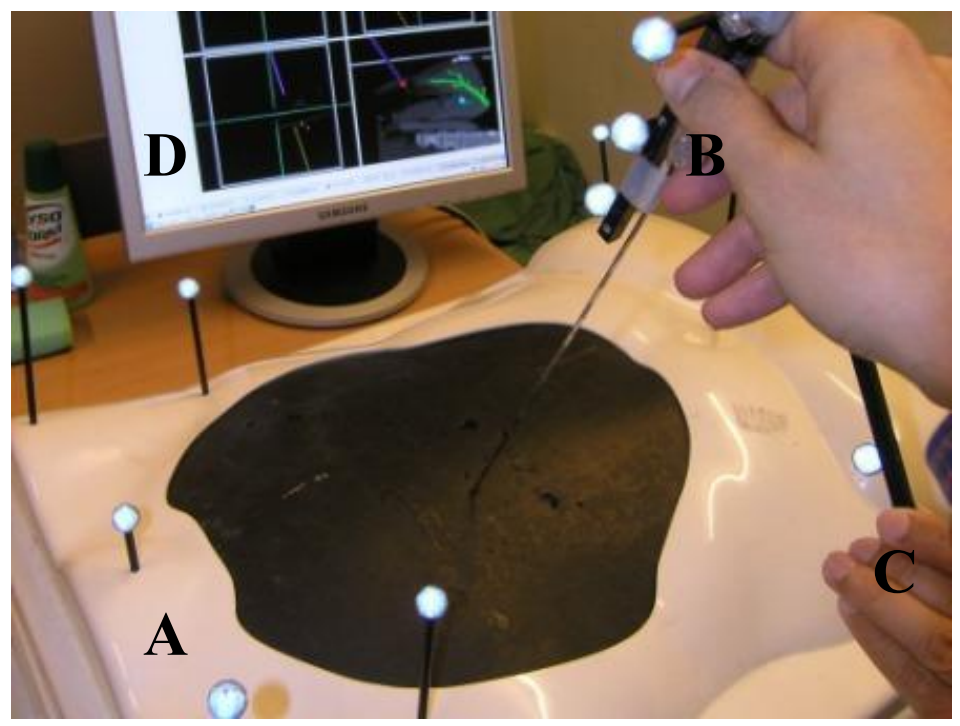

Fig. 5. ARIS*ER RFA system from the subjects view during setup and preparation for the user study: (A) Abdominal phantom; (B) RFA device (needle) with optical tracker; (C) pointing stick to chose external viewpoint (slightly hidden); (D) Flat screen TFT monitor (due to setup, window not in full screen modus).

\subsection{Study design}

First the subjects were asked to read the instructions on the experimental setup and the motivation of this study. Then they signed the informed consent form and personal information. The subjects rated their medical and technical expertise on a 5 -point-scale $(1=$ inexperienced, $5=$ experienced). Medical background was described with "general medical background" and use of medical images such as ultra sound (US) or computed tomography (CT) images. Technical background was described with "general engineering background" and use of virtual environments.

\subsection{Warm up phase}

The aim of the warm-up phase (ca. 5-25 minutes) was to introduce the subjects to the system and the visualization concepts as well as the different intra-operative tasks during the RFA procedures, which they have to fulfill. The intraoperative tasks for a needle placement have been described in words and instructed in the warm up phase as a 3-step task, adopted from [18]:

(1) Exploration and Search: Explore the abdomen and the liver and detect the target tumor (color coded). After completion of this task you should be able to describe the location of the liver in the abdomen and the location of the target tumor in the liver.

(2) Planning puncture and needle trajectory: Search for the appropriate attack point, the point where you want to place the puncture. Explore the needle trajectory, estimate the depth of the needle penetration and do risk estimation. Point with the needle tip at the place of puncture and indicate as accurate as possible your chosen trajectory line by targeting the center of the tumor. After completion of this you are ready to do the needle insertion. You should be able to describe the structures on the needle trajectory and have an idea of the length of the insertion path.

(3) Targeting and control of needle insertion: Insert the needle in the phantom. Insert the needle on the planned trajectory line and stop the needle tip as close as possible to the center of the tumor. After completion of this you are ready to do the final check and start the ablation.

After being introduced to this 3-step task, the subjects explored the functionalities of the system with the help of the study coordinators (TS, WA) and by performing the first two needle placements (grey and yellow tumor) one with the internal view and one ablation with the external view. The subjects were asked to follow the 3 -step task and refer to the study coordinator as soon as they felt unsure about anything. They were asked to finish the warm-up phase only when they feel "reasonably sure" about the tasks and the system. When the subjects felt ready for the test, they were asked to fill in the first part of the questionnaire. 


\subsection{Test phase}

Subjects were now asked to perform six needle placements (pink, blue, brown, purple, green, and red) three with each of the visualization concepts "as fast and as accurate as possible". Half of the subjects started with three placements with the internal view, half with the external view. They were asked to clearly indicate when they completed a step (3-step task). The study coordinators noted down time for completion of the different steps. The system recorded the location (tracking data) of the needle during the third step of the intra-operative procedure, from the moment of the puncturing to the final placement of the needle. The start-position was chosen to calculate the length of the planed/chosen needle path. Start and end of the recording were done manually by a study coordinator (WA). The subject was asked to hold the position at the final placement for some seconds. The nearest position of the needle tip to the tumor in the last three second of the needle placement has been calculated (accuracy of needle placement in $\mathrm{mm}$ ).

\subsection{Questionnaires}

All questions on usability were rated on Likert scale [9] - a psychometric response scale - from 0 "not at all" to 10 "very much". After the warm-up or learning phase, the subjects rated: How difficult was it to learn the different functions of the system e.g. CT views, 3D-view, trajectory lines? (learnability [12]) How much confident were you after the warm-up phase to fulfill the tasks successfully (before the six ablation simulation started)? Directly after each needle placement, the subjects had to rate their efficiency: How efficient (efficiency = time $x$ output, here accuracy) have you been at this needle placement? (subjective efficiency [12]) After three needle placements with the same visualization tool, the subjects had to rate: How much did the internal / external visualization support you during (1) exploration and search tasks (2) planning puncture and needle trajectory tasks (3) targeting and control of needle insertion? (general systems efficiency [12]) After the sixth needle placements with the same the subjects had to rate: How important was the $3 D$ view for you, the window in the down right corner? How much did you like it personally to use the tools (satisfaction, personal preference)? (satisfaction [12]) The last two questions should give feedback on the design goal of the visualization (see also Table 1, third colon): How good was the tool to check if the needle is still on the targeted trajectory line during insertion of the needle? How good was the tool to define the final placement of the needle (when to stop during insertion)? 


\section{RESULTS}

The subjects used the whole 5-point-scale from "inexperienced" to "experienced" both for medical and technical expertise. Average ratings for technical background were higher (mean $\pm \mathrm{SD}, 3.7 \pm 1.5)$ rated as medical background (2.7 \pm 1.3 ). All subjects background their professional background with "high" ( $>=4$ on a 5-point scale), the seven engineers with $4.6 \pm 1.5$, the three medical doctors with $4.3 \pm 1.9$. Only one subject was experienced in percutaneous needle placements.

\subsection{Usability questions}

After the learning and warm-up phase of 5-25 minutes, the subjects $(\mathrm{n}=10)$ rated the difficulty "to learn the different functions of the system" with $3.4 \pm 1.8$ (mean $\pm \mathrm{SD}$ ) on a 10-point-cale. Two persons rated it with "rather difficult" (6 and $7)$, the other eight rated the learnability [12] noticeable easier $(<5$, below the middle position of the scale). The "confidence to fulfill the tasks successfully" was rated with $6.2 \pm 1.5$, only one subject rated with $<5(3.5)$.

General task specific support "How much did the internal / external visualization support you during (1) exploration and search tasks (2) planning puncture and needle trajectory tasks (3) targeting and control of needle insertion?" was highly rated $7.1 \pm 2.0$ (mean $\pm \mathrm{SD}, \mathrm{n}=60)$, no differences have been found between external $7.08 \pm 1.9(\mathrm{n}=30)$ and internal 7.07 $\pm 2.1(\mathrm{n}=30)$ visualization concept. However different ratings have been given in with regard to the different tasks: Step one (exploration) was better supported $7.7 \pm 1.5(\mathrm{n}=20)$ than step two $6.9 \pm 2.0(\mathrm{n}=20)$, step three (insertion) had the lowest support $6.7 \pm 2.3(\mathrm{n}=20)$.

During the test the subjects had to rate their own efficiency. The mean self-rating of the efficiency was $6.4 \pm 1.9(\mathrm{n}=60)$ and showed no differences of the two concepts (external $6.6 \pm 2.1(\mathrm{n}=30)$ and internal $6.3 \pm 1.6(\mathrm{n}=30)$. The first placements with a new concept had significant lower efficiency ratings $(5.7 \pm 1.9, \mathrm{n}=20)$ as the third $(7.0 \pm 1.8, \mathrm{n}=20)$.

After the tests, the subjects also were asked to give a give feedback on the design goals of the visualization. The ratings for how good the tool is to "check if the needle is still on the targeted trajectory line during insertion of the needle" were for internal view $(7.7 \pm 1.5, \mathrm{n}=10)$ little higher as for external view $(7.0 \pm 1.5, \mathrm{n}=10)$. Also to "define the final placement of the needle" the internal view $(7.3 \pm 1.8, \mathrm{n}=10)$ was little higher rated as the external view $(6.8 \pm 1.3, \mathrm{n}=10)$.

\subsection{Time and accuracy of needle placement}

Overall time for 6 needle placements per subjects varied from 402s to 1330s, mean placement time for one tumor was $138 \pm 111 \mathrm{~s}(\mathrm{n}=59) .59$ out of 60 placements were "successful" only one needle placement failed or was given up after several attempts to plan the insertion. Manual timing succeeded in 137 out of 150 steps of the intra-operative tasks and for all successful needle placements (overall times for 3-steps).

The calculated accuracy of the final needle placement of the tracking data showed another picture: mean accuracy for all needle placements was $1.4 \pm 0.8 \mathrm{~cm}(\mathrm{n}=51)$. From 60 data sets 5 showed "impossible" tracking data and 4 were "empty". The remaining 51 data points can be rated as "a good estimation". The resulting mean penetration lengths (= puncture place to final position) was longest for the green tumor $(9.3 \pm 1 \mathrm{~cm})$ and shortest for the brown tumor $(2.2 \pm 0.6 \mathrm{~cm})$ seem to me quite realistic. Overall times (3-steps) $(p=.58)$, as well as placement accuracy $(p=.23)$ are correlating with the length of the needle path.

The calculated accuracies from the tracking data did not fully correspond with visual feedback the system gave. Approximately a third of all needle placements would be rated by subjects and study coordinators as "perfect hits" or "nearly perfect hits" (see Fig. 6). 


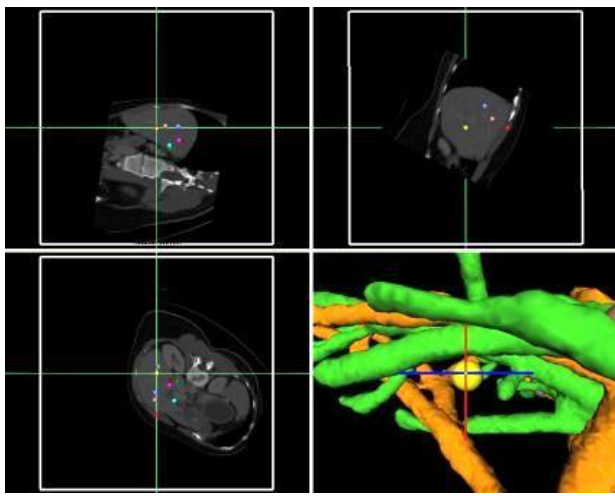

(a)
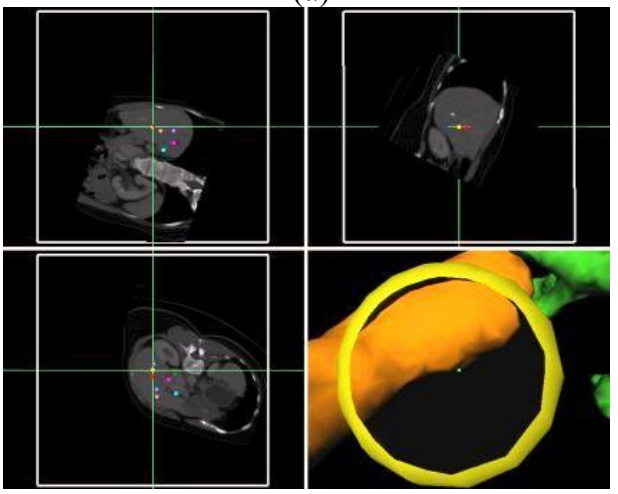

(c)

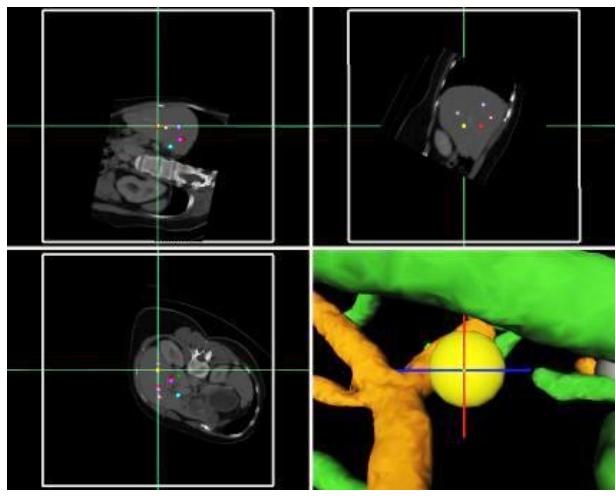

(b)
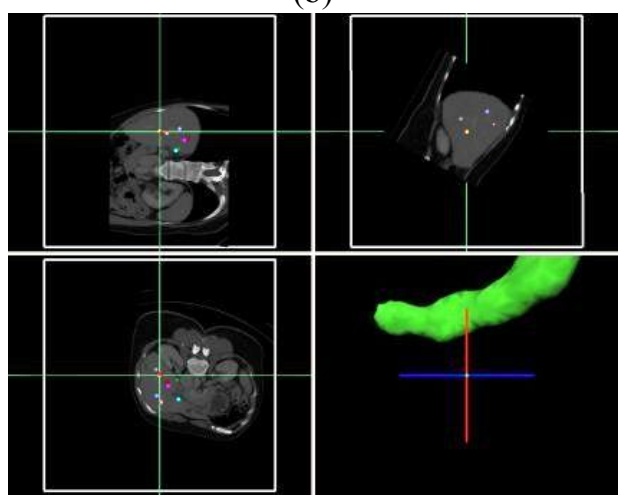

(d)

Fig. 6. "Perfect hit" - Needle placement into yellow tumor with internal 3D view (right down corner). (a) A suitable needle entry path is found. (b) Check if no big vessels are between puncture place and target. (c) Sphere of needle tip enters target yellow tumor. (d) The sphere of the needle tip totally masks the yellow sphere of the tumor. The system indicates "optimal needle placement accuracy".

\subsection{Observations and feedback from users}

Several subjects experienced - mostly in the in the warm-up phase - problems with unintentional rotation of the needle during insertion. The corresponding rotation of the coordinate system turns all images "up side down", this is such an unusual experience that it's not easy to keep orientation and do the necessary correction movements (rotation in the opposite way). This effect was especially irritating for the subject, when only focusing on one CT slice. In Fig. 6 from (c) to (d) you can easily recognize a rotation of $10-20 \mathrm{deg}$ in the internal horizontal view.

Some of the visualizations turned out to have too small dimensions for the actual working distance. The viewing distance to the monitor was approximately $1 \mathrm{~m}$. During the final placement of the needle you could easily observe subjects bending their head towards the screen. Especially for the CT slices an implemented zooming function would help the users for a better visibility of the visualizations and a more relaxed placement. 


\section{DISCUSSION}

We recruited subjects with medical and with technical background; although the two different specializations were clearly dividing the subjects in two groups, no differences on accuracy or efficiency between the two groups could be found. These findings could be explained by the small group size as well by the fact that the needle placement tasks were new or equally challenging for all subjects.

The system and its tools got a medium "learnability" rating [12]. This rating might be influenced not only by the system with its visualization tool but also by the intra-operative task (3-step-task). The self-ratings showed an (expected) learning effect in the first three needle placements but not in time or accuracy.

The systems efficiency (support) was highly ranked among all subjects with only five "lower" ratings $(<5)$ from two subjects. The tendency for better support in the first two tasks can be explained by the problem of the needle bending which is only relevant in the third step (insertion).

In general the feedback to the design was good. Only one subject rated three times below 5 . All other subjects used only positive ratings. This could be influenced by the fact that the test was done "on a prototype" what might imply "not mature technology". Subjects maybe do not dare to ask for (better) quality. We'll see in time and following studies if subjects that know (even better) what kind of functionality and services they can ask, become more daring in their demands [as discussed in 16].

On a general level our design and the results are in line with the findings from Seitel and colleagues [15]. They compared accuracy of needle placement with four different views (projection view, tool tip camera view, fixed camera view and 3D overview) on a phantom with simulated respiratory movements. They favored tool tip camera view and projection view for clinical use. Our data shows an effect of intra-operative tasks characteristics on subjective ratings of the user, as well as an effect of task complexities (e.g. depending on the tumor location). Those two aspects are highly relevant when transforming laboratory knowledge into a clinical setting.

The tendency of the needle to bend made the insertion task (third step) challenging for the subjects. Problems with the accuracy of the needle placements due to needle bending haven been reported in medical practice [13] as well as in relation with the accuracy of the tracking system [4]. Needle bending is not desirable for image-guided navigation. It affects tracking accuracy when needle tip is calibrated with respect to the reference frame fixed at the handle of the needle (opposite end). In our case we were simulating needle placements in virtual data with virtual targets and therefore did not affect placement accuracy. It can be seen as a side task for the user and a strengthening of the experimental setting. One important negative result of the needle bending is that we are at this moment not able to generate absolute placement accuracies.

The visualization tools are suitable for their aim. Problems have been encountered with the rotation of the needle as well as with the small dimensions and the reduced visibility of the tracked data. The usability of the tools will only be good when data and navigation aids are congruent [11], and when the viewpoint of the virtual information is optimized to the need of the user [19] in each of the intra-operative tasks.

The results show ambiguous results with beneficial and less favorable effects on user performance and usability of both visualization concepts depending on the characteristic of the intra-operative task (3-steps) and the tumor location (complexity of the task). The test could provide a lot of input for the next design step. Analyses of the data are still in an early phase. More insight is expected e.g. by analyzing videos taken from the subjects during the tests.

\section{ACKNOWLEDGEMENT}

The authors would like to thank all subjects of the ergonomic simulation study for their effort and time. Edvin van der Linden MD and Peter Pattynama MD (Erasmus MC, Rotterdam, the Netherlands) for their valuable expert user input for the design of the visualization tools. This research is done within the ARIS*ER project, which is funded by the European Union as part of the $6^{\text {th }}$ framework programme for research under the Marie Curie Actions for Human Resources and Mobility, contract MRTN-CT-2004-512400. 


\section{REFERENCES}

1 ARIS*ER project: http://www.ariser.info/

2 Dodd, III, G.D., Soulen, M.C., Kane, R.A., Livraghi, T., Lees, W.R., Yamashita, Y., Gillams, A.R., Karahan, O.I., and Rhim H. 2000. Minimally Invasive Treatment of Malignant Hepatic Tumors: At the Threshold of a Major Breakthrough, RadioGraphics 2000 20: 9-27.

Freudenthal, A., Samset, E., Gersak, B., Declerck, J., Schmalsieg, D., Casciaro, S., Rident, O. \& Vander Sloten, J. 2005. Augmented Reality in Surgery ARIS*ER, Research Training Network for Minimally Invasive Therapy Technologies, Endoscopic Review, Vol. 10, No. 23, 2005.

4 Howard, M.H., Nelson, R.C., Paulson, E.K., Kliewer, M.A., and Sheafor, D.H. 2001. An electronic device for needle placement during sonographically guided percutaneous intervention. Radiology 2001; 218: 905-11.

5 Hunt, E. \& Waller, D. 1999. Orientation and Wayfinding: A Review. [online available: http://citeseer.ist.psu.edu/cache/papers/cs/11224/http:zSzzSzdepts.washington.eduzSzhuntlabzSzvrzSzpubszSzhuntrevie w.pdf/hunt99orientation.pdf ]

6 Jalote-Parmar A, Pattynama PMT, Goossens RHM, Freudenthal A, Samset E, De Ridder H. 2006. Exploring a user centric methodology to investigate and integrate information gathered during medical intervention. In: Proceedings of the 16th World Congress on Ergonomics (IEA2006), Maastricht, the Netherlands, International Ergonomics Association. R.N. Pikaar, E.A.P. Koningsveld, and P.J.M. Settels (Eds), Elsevier Science.

7 Johnson, S., Healey, A., Evans, J., Murphy, M., Crawshaw, M., Gould, D. 2006. Physical and cognitive task analysis in interventional radiology. Clinical Radiology,61(1), pp. 97-103. [Cognitive tasks analysis online available: http://craive.org.uk/]

Kalkofen, D., Milko, S., Massoptier, L., Jalote-Parmar, A., and Risholm, P. 2007. ARIS*ER Demo Radio Frequency Ablation. Online available: $\mathrm{http}: / / \mathrm{www}$. ariser.info/projects/rfa_demo.php

9 Likert, R. 1932. A Technique for the Measurement of Attitudes. Archives of Psychology 140: 1-55.

10 McGahan, J.P. \& van Raalte, V.A. 2005. Tumor Ablation: Principles and Practice, History of Ablation. Springer Science+Business Media, Inc. 2005, 3-16.

11 Milgram, P., \& Colquhoun, H. 1999. A Taxonomy of Real and Virtual World Display Integration. Chapter 1 (1-16) in: Ohta Y., and Tamura H. (Eds) Mixed Reality - Merging Real and Virtual Worlds. Ohmsha (Tokyo) \& Springer Verlag (Berlin), 1999.

12 Nielsen, J. 1994, Usability Engineering, San Francisco: Morgan Kaufmann Publishers.

13 Poon, R.T., Kelvin K., Chi Ming Lam, Ai, V., Yuen, J., Sheung Tat Fan, \& Wong, J. 2004. Learning Curve for Radiofrequency Ablation of Liver Tumors - Prospective Analysis of Initial 100 Patients in a Tertiary Institution. Annals of Surgery, Volume 239, Number 4, April 2004. pp 441-449.

14 Schmalstieg, D., Fuhrmann, A., Hesina, G., Szalavari, Z., Encarnação, L. M., Gervautz, M., and Purgathofer, W. 2002. The Studierstube Augmented Reality Project. PRESENCE - Teleoperators and Virtual Environments, 11(1).

15 Seitel, A., Maier-Hein, L., Schawo, S., Radeleff, B.A., Mueller, S.A., Pianka, F., Schmied, B.M., Wolf, I., Meinzer, H.-P. 2007. In-vitro evaluation of different visualization approaches for computer assisted targeting in soft tissue. Int J CARS (2007) 2 (Suppl 1):S188-S190.

16 Stüdeli, T., Freudenthal, A., \& Samset, E. 2007a. Assessing Quality of Computer Systems and User Interfaces of Future Medical Workplaces - Augmented Reality for Minimally Invasive Therapy. Proceedings of UITQ 2007 Workshop May 20, 2007, Stockholm, Sweden.

17 Stüdeli, T., Freudenthal, A., \& H. de Ridder 2007b. Evaluation Framework of Ergonomic Requirements for Iterative Design Development of Computer Systems and their User Interfaces for Minimally Invasive Therapy. Proceeding of the Work With Computing Systems WWCS Conference, May 21st-24th 2007, Stockholm, Sweden.

18 Stüdeli, T., Kalkofen, D. \& Freudenthal, A. 2007. How do interventionists navigate to the tumour? In: C. Berlin and L-O. Bligård (Eds): Proceedings of the 39th Nordic Ergonomics Society Conference, Oct 1-3 2007, Lysekil, Sweden. Online available: http://www.nes2007.se/papers/A117_Studeli.pdf

19 Wang, W., \& Milgram, P. 2003. Effects of viewpoint displacement on navigational performance in virtual environments. Proc. 47st Annual Mtg Human Factors \& Ergonomics Society. Denver, 139-143, Oct. 2003. 